\title{
Intervene of EBV and HCMV in Relation to p27 and p73-Cell Regulatory Proteins in Histopathological Changes of Sinonasal and Nasopharyngeal Carcinomas
}

\author{
Ali JassimMM1', Mahmood MM1* and Mohammed Ali SH² \\ ${ }^{1}$ Department of Biology, College of Science, Mustansiriyah University, Iraq \\ ${ }^{2}$ Diseases Research Unit, Baghdad Medical College, University of Baghdad, Iraq \\ *Corresponding author: Majid Mohammed Mahmood, 1Department of Biology, College \\ of Science, Mustansiriyah University, Baghdad, Iraq; Email: \\ Majidmahmood93@yahoo.com
}

\section{Research article}

Volume 3 Issue 3

Received Date: July 03, 2019

Published Date: August 09, 2019

DOI: $10.23880 / v i j-16000217$

\section{Abstract}

Background: Sinonasal and Nasopharyngeal carcinomas are related to many environmental, genetic predisposing and viral infections. Many studies have implicated EBV in the progression of benign and malignant head and neck tumors by affecting cell cycle proteins expression. The p73 and p27 were implicated in carcinogenesis of nasopharyngeal and sinonasal carcinomas.

Objective: The research aimed to evaluate the impact of EBV and HCMV in relation to cellular protein expressions of p27 and p73-cell cycle regulatory genes on histopathological alterations of sinonasal and nasopharyngeal carcinomas.

Patients and Methods: Eighty three formalin fixed, paraffin embedded tissue blocks belong to 65 nasopharyngeal (NPC) and 18 sinonasal carcinomas (SNC) were investigated for EBV, HCMV, P73, P27 coexistences.

Results: The highest positivity percentage (50\%) of EBV in SNC was seen in moderately differentiated group. While in NPC tissues the highest level (74.5\%) of HCMV was detected in undifferentiated group. The highest positivity percentage (47.1\%) of p73 in SNC group was in moderate differentiated grade, while the highest positive-p27 percentage in NPC group was in undifferentiated grade $(76.3 \%)$.

Keywords: NPC: Nasopharyngeal Carcinoma; SNC: Sinonasal Carcinoma; HCMV: Human Cytomegalovirus; CISH: Chromogenic in Situ Hybridization; IHC: Immunohistochemical

\section{Introduction}

Nasopharyngeal carcinoma is a rare tumor; it accounts about $1 \%$ of all childhood malignancies [1]. A significant risk factors for NPC include environmental exposures, high consumption of salt-preserved fish, tobacco smoke in addition to infection with oncogenic viruses such as Epstein Barr Virus (EBV), which is most commonly associated with undifferentiated grades of NPC subtypes $[2,3]$. Epstein Barr virus also has been shown to be 


\section{Virology \& Immunology Journal}

associated to other human cancers, such as burkitt's lymphoma and Non-Hodgkin lymphoma etc [4,5]. Epstein Barr Virus encoded small RNAs (EBER1) which interacts with interferon-inducible protein kinase $R$ (PKR), inhibiting its activation and protecting infected cells from interferon (IFN) induced apoptosis and induce the expression of growth factors that promote cell survival $[6,7]$.

Human cytomegalovirus (HCMV) infection might have role in building up the tumor cells through protection of certain tumor cells from apoptosis and modulating angiogenesis through latent HCMV infection that promote ability of tumor cells to evade immune response [8]. At least four protein encoded by Unique Short (US) region of HCMV genome involved in inhibition of MHC-1 expression, either by acting directly on MHC-1 or acting on MHC-1 associated proteins, such as transporter associated with antigen processing (TAP) and tapasin [9].

The deletion or silencing of p73 gene in pancreatic cancers, neuroblastoma, breast and hepatocellular carcinomas suggesting its potential role as tumor suppressor gene [10]. The p27 levels in human tumors may vary as a result of posttranscriptional and posttranslational regulation [11]. In addition, variable expression of $\mathrm{p} 27^{\mathrm{kip} 1}$ protein was found to be excessively associated with cell proliferation which is linked to many types of human tumors, such as nasopharyngeal carcinomas, oral cavity squamous cell carcinoma (OSC), gastric cancer, and small cell lung cancer [12]. Moreover, p27 dysregulation was found to play a role both in cell cycle dysregulation and chromosomal instability, leading to higher-grade NPC malignancy and an increased cell proliferation in sinonasal tumors [13].

The aim of this study is to analyze the rate of accompaniment of P27 and p73-gene translational expression with EBV and HCMV in nasopharyngeal and sinonasal malignant tumors.

\section{Materials and Methods}

This study has enrolled a total number of eighty three (83) selected formalin-fixed, paraffin-embedded blocks from nasopharyngeal and sinonasal tissues. These tissue blocks were collected from histopathological laboratories of several hospitals in Baghdad-Iraq which included 18 sinonasal and 65 nasopharyngeal carcinomas. The diagnoses were based on their accompanied pathological reports of the corresponding patients. They were 55 male and 28 female whose age range was between (17-83) years.
The detection of HCMV, EBV, P27 and P73 were carried out in the Clinical Communicable Diseases Research Unit (CCDRU) / College of Medicine/ University of Baghdad while the histopathological preparations of tissues blocks was done in the histopathological laboratories of Specialized Surgeries Hospital / Medical City.

The detection of EBV and HCMV was performed by using Chromogenic in situ hybridization (CISH) reactions kit (purchased from ZytoVision/ Germany) on $5 \mu \mathrm{m}$ tissue sections with the aid of digoxigenin-labeled oligonucleotides probe which targets EBV-EBERs and HCMVpp65 DNA (Cat. Number: T-1114-400 and T-1113-400).

The detailed methods for performing CISH reactions were conducted according to the instructions of the above manufacturing company. Immunohistochemical (IHC) assays were used to evaluate p73 and p27 expression in SNC and NPC tissues by using Monoclonal Rabbit Anti-p27 and Anti-p73 antibodies (Cat. Numbers: ab40658 and ab32034) and rabbit specific HRP/DAB detection IHC Kit, Abcam /UK) that targeted nuclear specific proteins. The quantification of CISH and IHC signals was assessed under light microscopy at (100X). Scoring and intensity of signals were done according to [14].

\section{Statistical Analysis}

In this study, SPSS program (version-21) was used for statistical analysis where Chi-Square test $(\chi 2)$ was used to assess the significances between variables.

\section{Results}

The results of co-associations of EBV, HCMV, p27 and p73 in relation to SNC and NPC grading were detailed in (Table 1) and (Figure 1 and 2). The positive-EBV-CISH signaling results in relation to the examined histopathological grading of SNC showed that $(50 \%)$ of these tissues revealed moderately differentiated grade. On the other hand, it was found that $(73.2 \%)$ of positiveEBV signaling in NPC tissues revealed undifferentiated grade.

The highest positivity percentage (50\%) of HCMV in SNC group was noticed in poorly differentiated group whereas in NPC the highest HCMV-positivity was in undifferentiated group (74.5\%).

In regard to p27 expression in SNC group, the poorly differentiated group showed the highest value which was $(41.7 \%)$, while $(76.3 \%)$ (29 out of total positive 38 


\section{Virology \& Immunology Journal}

tissues) of NPC tissues were graded as undifferentiated as $\quad$ seen in Figure 1.
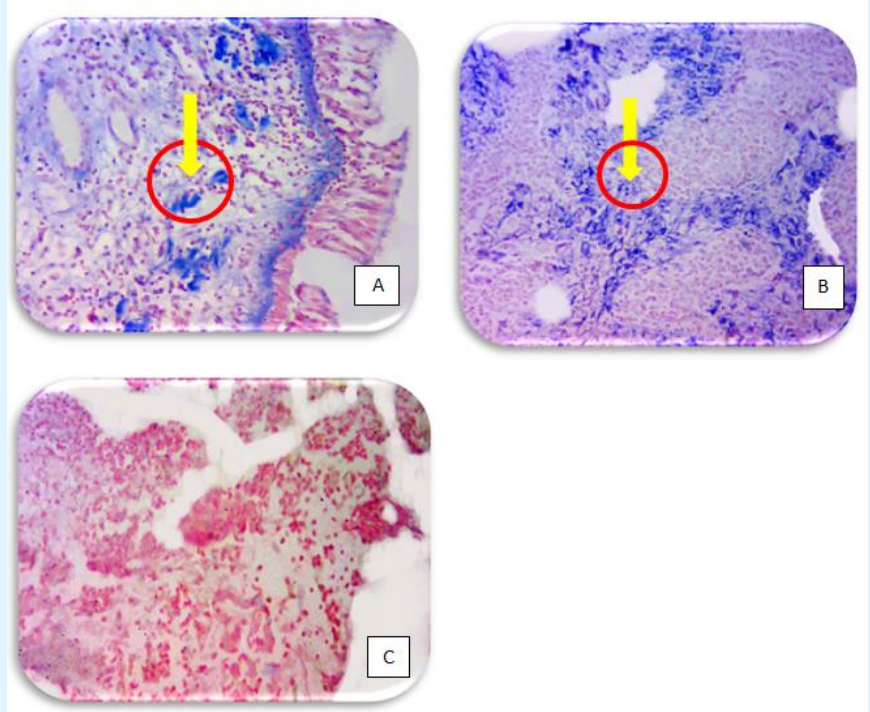

Figure 1: Microscopic appearance of EBV and HCMV-CISH signals in nasopharyngeal and sinonasal carcinomas. Blueviolet signals are observed at the site of complementary sequence in the cell's nuclei. A: Carcinoma with high score EBV and moderate intensity reactions (10X). B: High score HCMV and moderate intensity reactions (10X). C: Carcinoma with negative reaction (10X).

However, table (1) illustrates that the highest level of p73 expression was (47.1\%) in SNC moderately differentiated groups as noticed in Figure 2 whereas, among those forty seven positive results of P73-IHC

reactions, undifferentiated groups revealed the highest rates 35/47 (74.5\%). Even though there were no statistical differences among groups regarding EBV, HCMV, p27 and p73 in different grades ( $>>0.05)$.
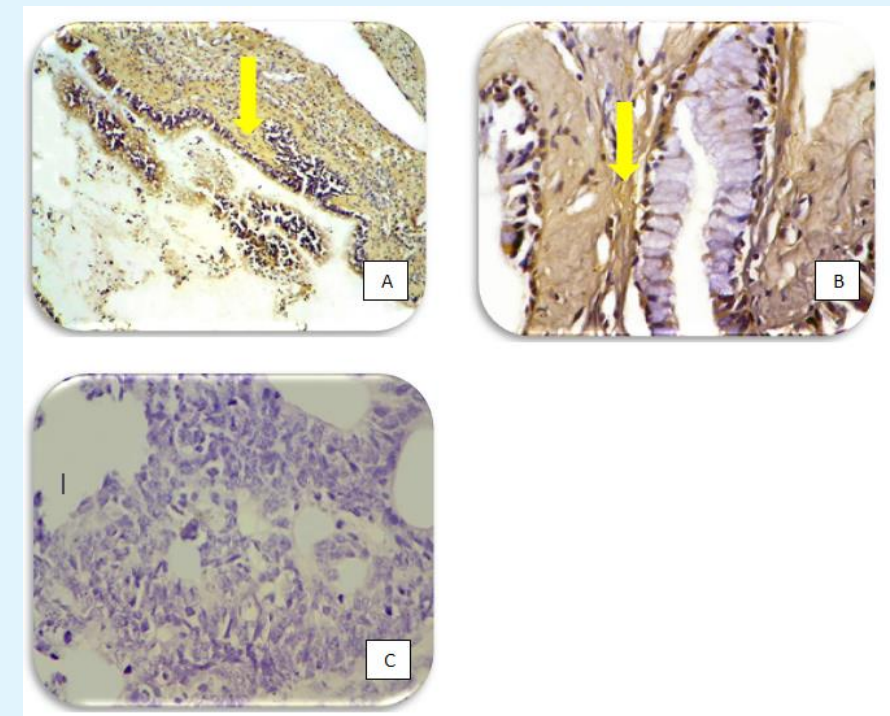

Figure 2: Microscopic photograph of IHC-p27 and p73 positive signals appeared as a brown discoloration (yellow arrow) at nuclear and cytoplasmic location in nasopharyngeal and sinonasal carcinoma. A: P27 positive staining in carcinoma tissues infected with EBV and HCMV (10X) B: P73 positive staining in carcinoma tissues infected with EBV and HCMV (10X) C: Carcinoma with negative staining (40X). 


\section{Virology \& Immunology Journal}

\begin{tabular}{|c|c|c|c|c|c|c|c|c|c|c|}
\hline \multirow{3}{*}{ 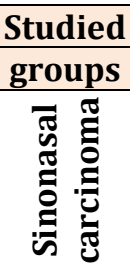 } & \multirow{2}{*}{\multicolumn{2}{|c|}{ Diagnosis }} & \multicolumn{2}{|c|}{ EBV } & \multicolumn{2}{|c|}{ HCMV } & \multicolumn{2}{|c|}{ P27 } & \multicolumn{2}{|c|}{ P73 } \\
\hline & & & Positive & Negative & Positive & Negative & Positive & Negative & Positive & Negative \\
\hline & \multirow[t]{2}{*}{ M.D. } & $\mathrm{N}$ & 8 & 0 & 4 & 4 & 4 & 4 & 8 & 0 \\
\hline \multirow[t]{7}{*}{ ర్త } & & $\%$ & $50 \%$ & $0 \%$ & $33.30 \%$ & $66.70 \%$ & $33.30 \%$ & $66.70 \%$ & $47.10 \%$ & $0 \%$ \\
\hline & \multirow{2}{*}{ P.D. } & $\mathrm{N}$ & 5 & 2 & 6 & 1 & 5 & 2 & 6 & 1 \\
\hline & & $\%$ & $31.20 \%$ & $100 \%$ & $50 \%$ & $16.70 \%$ & $41.70 \%$ & $33.30 \%$ & $35.30 \%$ & $100 \%$ \\
\hline & \multirow{2}{*}{ U.D. } & $\mathrm{N}$ & 3 & 0 & 2 & 1 & 3 & 0 & 3 & 0 \\
\hline & & $\%$ & $18.80 \%$ & $0 \%$ & $16.70 \%$ & $16.70 \%$ & $25 \%$ & $0 \%$ & $17.60 \%$ & $0 \%$ \\
\hline & \multirow{2}{*}{$\mathrm{T}$. } & $\mathrm{N}$ & 16 & 2 & 12 & 6 & 12 & 6 & 17 & 1 \\
\hline & & $\%$ & $100 \%$ & $100 \%$ & $100 \%$ & $100 \%$ & $100 \%$ & $100 \%$ & $100 \%$ & $100 \%$ \\
\hline \multicolumn{3}{|c|}{ Pearson Chi-Square } & \multicolumn{2}{|c|}{$\mathrm{P}=0.171$} & \multicolumn{2}{|c|}{$\mathrm{P}=0.343$} & \multicolumn{2}{|c|}{$\mathrm{P}=0.276$} & \multicolumn{2}{|c|}{$\mathrm{P}=0.435$} \\
\hline \multicolumn{3}{|c|}{ (P-value) } & \multicolumn{2}{|c|}{ Non Sign $(\mathrm{P}>0.05)$} & \multicolumn{2}{|c|}{ Non Sign. $(\mathrm{P}>0.05)$} & \multicolumn{2}{|c|}{ Non Sign $(\mathrm{P}>0.05)$} & \multicolumn{2}{|c|}{ Non Sign. $(\mathrm{P}>0.05)$} \\
\hline \multirow{10}{*}{ 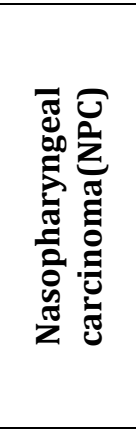 } & \multirow{2}{*}{ W.D. } & $\mathrm{N}$ & 10 & 0 & 8 & 2 & 7 & 3 & 8 & 2 \\
\hline & & $\%$ & $17.90 \%$ & $0 \%$ & $15.70 \%$ & $14.30 \%$ & $18.40 \%$ & $11.10 \%$ & $17 \%$ & $11.10 \%$ \\
\hline & \multirow{2}{*}{ M.D. } & $\mathrm{N}$ & 1 & 1 & 1 & 1 & 0 & 2 & 0 & 2 \\
\hline & & $\%$ & $1.80 \%$ & $11.10 \%$ & $2 \%$ & $7.10 \%$ & $0 \%$ & $7.40 \%$ & $0 \%$ & $11.10 \%$ \\
\hline & \multirow{2}{*}{ P.D. } & $\mathrm{N}$ & 4 & 0 & 4 & 0 & 2 & 2 & 4 & 0 \\
\hline & & $\%$ & $7.10 \%$ & $0 \%$ & $7.80 \%$ & $0 \%$ & $5.30 \%$ & $7.40 \%$ & $8.50 \%$ & $0 \%$ \\
\hline & \multirow{2}{*}{ U.D. } & $\mathrm{N}$ & 41 & 8 & 38 & 11 & 29 & 20 & 35 & 14 \\
\hline & & $\%$ & $73.20 \%$ & $88.90 \%$ & $74.50 \%$ & $78.60 \%$ & $76.30 \%$ & $74.10 \%$ & $74.50 \%$ & $77.80 \%$ \\
\hline & \multirow{2}{*}{$\mathrm{T}$} & $\mathrm{N}$ & 56 & 9 & 51 & 14 & 38 & 27 & 47 & 18 \\
\hline & & $\%$ & $100 \%$ & $100 \%$ & $100 \%$ & $100 \%$ & $100 \%$ & $100 \%$ & $100 \%$ & $100 \%$ \\
\hline \multicolumn{3}{|c|}{ Pearson Chi-Square } & \multicolumn{2}{|c|}{$\mathrm{P}=0.196$} & \multicolumn{2}{|c|}{$\mathrm{P}=0.553$} & \multicolumn{2}{|c|}{$\mathrm{P}=0.322$} & \multicolumn{2}{|c|}{$\mathrm{P}=0.071$} \\
\hline & P-value & & Non Sigl & $(\mathrm{P}>0.05)$ & Non Sign & $(\mathrm{P}>0.05)$ & Non Sign & $(\mathrm{P}>0.05)$ & NonSign & $(\mathrm{P}>0.05)$ \\
\hline
\end{tabular}

Table 1: Interplay effects of EBV \& HCMV with cell cycle regulatory proteins (p27 and p73) in SNC and NPC grading. MD: Moderately differentiated, PD: Poorly Differentiated, UD: Undifferentiated WD: Well differentiated, T: Total. Correlation of EBV and HCMV-CISH with p27 and p73 in Patients with Sinonasal and Nasopharyngeal Malignant Tumors.

The result show weak negative non-significant $(\mathrm{P}>$ 0.05) relationships between EBV and HCMV with p73 scores in NPC group, while this relationship was a weak positive in SNC as well as there are weak negative relationships don't reach a significant level $(\mathrm{P}>0.05)$ between EBV and p27 scores in NPC group and positive in SNC while this relationship was a weak positive nonsignificant $(\mathrm{P}>0.05)$ between HCMV and p27 score in NPC and negative in SNC (Table 2).

\begin{tabular}{|c|c|c|c|c|c|}
\hline \multirow{2}{*}{ Spearman's rho (Scoring) } & \multicolumn{2}{|c|}{ P27 } & \multicolumn{2}{c|}{ P73 } \\
\cline { 3 - 6 } \multicolumn{2}{|c|}{} & $\begin{array}{c}\text { Nasopharyngeal } \\
\text { carcinoma }\end{array}$ & $\begin{array}{c}\text { Sinonasal } \\
\text { carcinoma }\end{array}$ & $\begin{array}{c}\text { Nasopharyngeal } \\
\text { carcinoma }\end{array}$ & Sinonasal carcinoma \\
\hline \multirow{2}{*}{ EBV } & r. & -.027 & .329 & -.029 & .423 \\
\cline { 2 - 6 } & P-value & .830 & .183 & .821 & .080 \\
\hline \multirow{2}{*}{ HCMV } & r. & 0.068 & -0.339 & -0.133 & .3020 \\
\cline { 2 - 6 } & P-value & 0.593 & 0.168 & .2910 & .2220 \\
\hline
\end{tabular}

Table 2: Spearman's rho statistical testing to evaluate studied molecular markers scoring in relation to EBV\& HCMV infections in sinonasal and nasopharyngeal lesions.

Correlation is non-significant at the $(\mathrm{P}>0.05)$ level. 


\section{Discussion}

In regards to oncogenesis, viral agents could play a role in tissue carcinogenesis/oncogenesis at either an early or late or continuously from early to late events. There is a scarcity of literatures concerned with the relation of HCMV to different grades of NPC and SNC. A possible correlation between HCMV infection with NPC has been suggested by Desgranges C, et al., Suliman RSAG, et al. and Ahmed $H$ [15-17]. Herein, in this study, it was found that the grade of HCMV-related SNC and NPC consistently elevated in later stages of disease comprising (66.7\% and $82.3 \%$ ) in SNC and NPC, respectively. These results showed that virus could played a role in late events of the multistep of sinonasal and nasopharyngeal carcinogenesis.

These findings provide a useful indication that certain types of cancers are infected with HCMV and then trigger the oncogenic properties as progression and invasiveness of such cancer cells. The viral gene products of CMV can dysregulate cellular pathways involved in mutagenesis, apoptosis, cell cycle, oncogenesis and cell invasion as reported by Mittal SK, et al. and Kumari P, et al. $[18,19]$.

Human cytomegalovirus and tumors are considered two players for one goal represented by escape from immune response. HCMV is a latent herpesvirus that maintains dynamic relationships with the immune system so as to help the tumor cell to evade the first line of host defense and induce a state of immune tolerance in which it can grow [20]. In addition, HCMV infection have been related to the further development of SNC and NPC carcinomas and possibly has exerted its oncogenic influences in concert with co-factors in collaboration with this viral infection. It can be concluded that the high rates of HCMV in their evidently correlation with the grading of SNC and NPC could point for a molecular role for HCMV in the etiology of these cancers which could probably occur late, along with other important oncogenic viruses. More extensive studies along with inclusion of high cumulative numbers to unravel their exact role in end SNC and NPC oncogenesis.

In addition, the positive-EBV results recorded in NPC illustrates that the virus has a role as a late events in NPC carcinogenesis process acting in an interplay manner with many other molecular factors because the highest percentage of viruses was found in the advanced grade of NPC which constituted (80.3\%).

The higher results of EBER in present study of tumor groups are supported by other studies which found the

\section{Virology \& Immunology Journal}

percent of EBER in NPC were (81\%, 91\% and 62.2\%), respectively [21-23]. It is noteworthy in this study that an increasing trend of association of EBV infection which accompanied the deterioration in the histopathological features of the examined NPC cancer tissues, that is an increasing percentage of detection of EBV with the advancing of grading of cancerous tissues of this study. This could also means, in turn, that there are additional possible effects of EBV infection, along with other factors, in deterioration the histopathology of NPC cancerous tissues obtained from those studied patients.

The high rate of EBV infections as well as their evident correlation with the differentiation of SNC and NPC could point to the role of EBV in these cancers as a molecular attack which probably occurred at an early or a late event (respectively), along with other important oncogenic viruses.

It was revealed that EBV is an oncogenic virus implicated in many neoplastic diseases, notably lymphomas and NPC and its presence in tumor tissues represents its real participation in carcinogenesis [24,25]. Herein, we could speculate that this virus might encourage or accelerate the infected nasal tissues towards cancerous ones (along with other factors in the carcinogenesis process). Also these findings could be explained that EBV infections with high viral load and high transcriptional and post-translational process of active viral genes that enhance changes or genetic alteration of cells and histological features of NP and SN tissues.

The findings of positive-p27 marker in association with SNC grade showed that the percentages of p27 in a late disease stage was (66.7\%) so that this gene expression has role at a late molecular events of sinonasal carcinogenesis acting in an interplay manner with other molecular factors.

In regards to NPC, the results of $\mathrm{p} 27$ were higher at a late stage of disease (81.6\%) which could explain that the p27 play an important role as a late event of nasopharyngeal oncogenesis in collaboration with some other factors.

On the other hand, positive-p73 marker in association with SNC grades are pointing to the effect of p73 which was mainly as an early events and continued (although to a lesser effect) as a late event in sinonasal carcinogenesis along with the role of other viruses as well as many other factors in this process. Whereas, p73 in NPC tissue groups have a late role in nasopharyngeal carcinogenesis in 


\section{Virology \& Immunology Journal}

association with the roles of many other factors in this process.

\section{Conclusion}

The co-infections of EBV and HCMV may participate together in sinonasal and nasopharyngeal carcinogenesis during its late eventual stages. The high expression rates of p27 and p73 protein expression in malignant nasopharyngeal tissues with limited or even no influential regulatory role, may indicate mutational events in such proteins.

\section{References}

1. Wu L, Li C, Pan L (2018) Nasopharyngeal carcinoma: a review of current updates. Experimental Therapeutic Medicine 15(4): 3687-3692.

2. Lee HM, Lo KW, Wei W, Tsao SW, Chung GTY, et al. (2017) Oncogenic S1P signalling in EBV-associated nasopharyngeal carcinoma activates AKT and promotes cell migration through $\mathrm{S} 1 \mathrm{P}$ receptor 3 . J Pathol 242(1): 62-72.

3. Ma BB, Hui EP, Chan AT (2017) Investigational drugs for nasopharyngeal carcinoma. Expert opinion on investigational drugs 26(6): 677-685.

4. Li YQ, Khin NS, Chua ML (2018) The evolution of Epstein-Barr virus detection in nasopharyngeal carcinoma. Cancer Biology Medicine 15(1): 1-5.

5. Tsang CM, Tsao SW (2015) The role of Epstein-Barr virus infection in the pathogenesis of nasopharyngeal carcinoma. Virologica Sinica 30(2): 107-121.

6. Agnieszka S (2016) Genetic analysis of the role of Epstein-Barr virus nuclear antigen leader protein (EBNA-LP) in B cell transformation. PhD. thesis, Department of Medicine, Imperial College London, UK, pp: 40.

7. Fitzsimmons L, Kelly G (2017) EBV and apoptosis: the viral master regulator of cell fate? Viruses 9(11): 339 $-373$.

8. Biolatti M, Dell'Oste V, Pautasso S, Gugliesi F, von Einem J, et al. (2018) Human cytomegalovirus tegument protein pp65 (pUL83) dampens type I interferon production by inactivating the DNA sensor cGAS without affecting STING. J virol 92(6): e0177417.
9. Hansen TH, Bouvier M (2009) MHC class I antigen presentation: learning from viral evasion strategies. Nature Reviews Immunol 9(7): 503-513.

10. Vélez Cruz R, Johnson D (2017) The retinoblastoma (RB) tumor suppressor: pushing back against genome instability on multiple fronts. Int J Molecular Sci 18(8): 1776-1792.

11. Sekiya T, Bronstein MD, Benfini K, Longuini VC, Jallad RS, et al. (2014) p27 variant and corticotropinoma susceptibility: a genetic and in vitro study. Endocrinerelated cancer 21(3): 395-404.

12. Wangefjord S, Brändstedt J, Lindquist KE, Nodin B, Jirström K, et al. (2013) Associations of beta-catenin alterations and MSI screening status with expression of key cell cycle regulating proteins and survival from colorectal cancer. Diagnostic Pathol 8(1): 10-23.

13. Patel P, Tsiperson V, Gottesman SR, Somma J, Blain SW (2018) Dual inhibition of CDK4 and CDK2 via targeting p27 tyrosine phosphorylation induces a potent and durable response in breast cancer cells. Molecular Cancer Res 16(3): 361-377.

14. Zlobec I, Steele R, Michel RP, Compton CC, Lugli A, et al. (2006) Scoring of p53, VEGF, Bcl-2 and APAF-1 immunohistochemistry and interobserver reliability in colorectal cancer. Modern pathol 19(9): 12361242.

15. Desgranges C, Seigneurin J, Baccard M, Nejmi S (1983) Cytomegalovirus Isolations From Cell Cultures Derived From Epstein-Barr Virus-Associated Nasopharyngeal Carcinoma. J National Cancer Institute 71(1): 39-43.

16. Suliman RSAG, El Aziz MS, Ahmed HG (2014) Relationship between Cytokeratins expression and Human Herpes Viruses (EBV, CMV, HSV) infections in Nasopharyngeal Carcinoma. Int J Sci Res 3(10): 21202123.

17. Ahmed H, Suliman RSA, Ibraheem M, Ashankyty IM, Albieh ZA, et al. (2018) Role of human Cytomegalovirus in the etiology of nasopharyngeal carcinoma. J Cancer Res Ther 14(3): 583-586.

18. Mittal SK, Roche PA (2015) Suppression of antigen presentation by IL-10. Current opinion Immunol 34(1): 22-27. 


\section{Virology \& Immunology Journal}

19. Kumari P, Saha I, Narayanan A, Narayanan S, Takaoka A, et al. (2017) Essential role of HCMV deubiquitinase in promoting oncogenesis by targeting anti-viral innate immune signaling pathways. Cell Death Disease 8(10): 3078-3084.

20. Lepiller Q, Abbas W, KumarA, Tripathy MK, Herbein G (2013) HCMVactivatesthe IL-6-JAK STAT3 axis in HepG2 cells and primary human hepatocytes. PLoSOne 8(3): e59591.

21. Bar Sela G, Kuten A, Minkov I, Gov Ari E, Ben Izhak O (2004) Prevalence and relevance of EBV latency in nasopharyngeal carcinoma in Israel. J Clinical Pathol 57(3): 290-293.

22. Chen HL, Peng J, Zhu XB, Gao J, Xue JL, et al. (2010) Detection of EBV in nasopharyngeal carcinoma by quantum dot fluorescent in situ hybridization. Experimental Molecular Pathol 89(3): 367-371.

23. Saikia A, Raphael V, Shunyu NB, Khonglah Y, Mishra J, et al. (2016) Analysis of Epstein Barr Virus Encoded RNA Expression in Nasopharyngeal Carcinoma in North-Eastern India: A Chromogenic in Situ Hybridization Based Study. Iranian J Otorhinolaryngol 28(87): 267-274.

24. Esau D (2017) Viral causes of lymphoma: the history of Epstein-Barr virus and human T-lymphotropic virus 1. Virology: Res Treatment 25(8): 1-5.

25. Vranic S, Cyprian FS, Akhtar S, Moustafa A (2018) The role of Epstein-Barr virus (EBV) in cervical cancer: A brief update. Frontiers Oncology 8(113): 1-8. 